\title{
Über den Einfluss der Farbenempfindungen auf die Sinnesfunctionen.
}

\author{
Von
}

Victor Urbantschitseh in Wien.

Über den Einfluss, den die versehiedenen Farbenempfindungen auf den Hörsinn und statischen Sinn, auf den Geschmack und Geruch sowie auf den Tastsinn zu nehmen vermögen, habe ich bereits vor längerer Zeit berichtet ${ }^{1}$ ). Erneute Untersuchungen, die ich darüber anstellte, lieferten mir weitere, wie ich glaube, bemerkenswerte Beobachtungen, weshalb ich im Nachfolgenden eine zusammenfassende Darstellung dieses Gegenstandes gebe.

Beim Durchsehen durch ein farbiges Glas oder beim Anblicke eines Farbenfeldes treten bei manchen Personen, je nach der auf das Auge einwirkenden Farbe, Veränderungen in der Stärke der verschiedenen Sinnesempfindungen ein, in der Weise, dass einzelne Sinnesempfindungen durch gewisse Farben gesteigert, durch andere herabgesetzt werden, während sich wieder einzelne Farben als wirkungslos erweisen. Von den in gleichem Sinne einwirkenden Farben (empfindungssteigernd oder -herabsetzend) besitzen nicht alle einen gleich grossen Einfluss, sondern bei einzelnen Farben tritt die Änderung der Empfindungsstärke besonders auffällig, bei anderen Farben nur in geringem Grade hervor. Zuweilen wirken sämmtliche Farben auf eine oder auf sämmtliche Sinnesempfindungen in gleichem Sinne (steigernd oder herabsetzend) ein und weisen nur Unterschiede in der Stärke ihrer Einwirkung auf; ein andermal ergeben eine oder einzelne Farben eine Änderung der Sinnesempfindung, während sich die übrigen Farben indifferent verhalten. Bei anderen, besonders bei schwer erregbaren Personen, findet überhaupt keine Beeinflussung der Sinnesempfindungen durch Farbeneinwirkungen statt. Werden die einzelnen Farbengläser oder Farbenfelder nur dem einen Auge

1) Pflüger's Archiv Bd. 42 S. 161 u. f. 1888, Bd. 94 S. 385 u. f. 1903. Zeitschr. f. Ohrenheilk. Bd. 31 S. 257. 1897.

E. Pflüger, Archiv für Physiologie. Bd. 106. 
vorgesetzt, so entsteben die der jedesmaligen Farbe zukommenden Veränderungen in der Stärke der Sinnesempfindungen manchmal nur auf der gleichseitigen Körperhälfte. Vergleichsweise mit dem rechten und linken Auge vorgenommenen Versuche lehren, dass ein und dieselbe Farbe häufig an der rechten und linken Körperhälfte übereinstimmende Intensitätsveränderungen der Sinnesempfindungen ergibt, zuweilen aber darin Unterschiede aufweist, ja, in einzelnen Fällen sogar ein entgegengesetztes Verhalten erkennen lässt. So vermag eine bestimmte Farbe, bei ihrer Einwirkung auf das rechte Auge allein, auf der rechten Körperseite eine Steigerung, vom linken Auge aus dagegen auf der linken Körperseite eine Abschwächung einer Sinnesempfindung hervorzurufen, während wieder ein andermal eine Beeinflussung der Sinnesempfindung nur auf einer und nicht auch auf der anderen Körperseite hervortritt. Auffälliger Weise entspricht der bei monokulärer Farbeneinwirkung erscheinende Einfluss auf die Sinnesempfindungen nicht immer dem bei binokulärer Farbeneinwirkung (s. später).

Mitunter beeinflusst eine monokuläre Farbeneinwirkung nicht die Sinnesempfindung der entsprechenden, sondern der entgegengesetzten Seite, wobei eine solche gekreuzte Einwirkung vom rechten und linken Auge aus oder nur von einem Auge aus erfolgt (s. später).

Der Einfluss, den eine bestimmte Farbe auf den einen Sinn aufweist, besteht, wie später noch näher erörtert wird, nicht immer auch für die übrigen Sirnesempfindungen, so wie auch dieselbe Sinnesempfindung an verschiedenen Tagen, bei sonst gleicher Versuchsanordnung, ein verschiedenes Verhalten anfweisen kann. Allerdings zeigten die meisten meiner Versuchsfälle bei einer stark ausgesprochenen Beeinflussbarkeit einer Sinnesempfindung durch eine bestimmte Farbe die gleiche Art der Beeinflussung auch an den übrigen Sinnesempfindungen und an den verschiedenen Versuchstagen.

Bezüglich der einzelnen Sinne ergaben meine Untersuchungen nit den verschiedenen Farbenempfindungen Folgendes:

\section{Einfluss der Farbenempfindungen auf den Hörșinn.}

Die Farbenempfindungen vermögen die Hörschärfe, das qualitative Hören, die subjective Localisation der Tonempfindungen und die subjectiven Gehörsempfindungen zu beeinflussen.

a). Die Hörschärfe erfährt durch die verșchiedenen Farbenempfindungen bald eine Steigerung, bald eine Herabsetzung, wobei 
dieselbe Farbe, je nach der Versuchsperson, das Hörvermögen entweder steigert oder vermindert oder aber unbeeinflusst lässt.. Die durch Farbenempfindungen hervorgerufenen Veränderungen der Hörschärfe sind zumeist nicht bedeutend, und es empfiehlt sich daher, zu diesen Versuchen nur sehr schwache Schalleinwirkungen zu verwenden, da sich an der Hörgrenze bereits geringe Hörschwankungen deutlich bemerkbar machen.

Unter zehn Versuchsfällen bewirkte Rot sechsmal eine Steigerung, zweimal eine Herabsetzung, zweimal keine Änderung der Hörschärfe, Grün viermal eine Steigerung, fünfmal eine Herabsetzung, einmal keine Änderung, Gelb dreimal eine Steigerung, fünfmal eine Herabsetzung, zweimal keine Änderung, Blau zweimal eine Steigerung, viermal eine Herabsetzung, viermal keine Änderung, Violett zweimal eine Steigerung, fünfmal eine Herabsetzung, dreimal keine Änderunğ. In einem dieser zehn Fälle fand eine Gehörssteigerung durch alle Farbenempfindungen statt, am meisten durch Gelb, am wenigsten durch Blau und Violett, in einem anderen Falle eine Herabsetzung dẹr Hörschärfe durch alle Farben, besonders durch Blau. In einem Falle verhielt sich Rot, in einem anderen Gelb indifferent, indes die übrigen Farben (einmal besonders Blau; ein andermal Violett) eine Herabsetzung der Hörschärfe ergaben. In einem Falle erregte. Blau eine Hörsteigerung, indes die übrigen Farben indifferent blieben. Bei Prüfung mit dem Uhrticken, das aus zwei ungleichen Schlägen besteht, verschwindet zuweilen bei manchen Farbeneinwirkungen der schwächere Schlag vollstäudig, während der stärkere Schlag abgescbwächt vernommen wird. Bei einer Versuchsperson verminderte Grün das Hören beider Schläge, Blau hob das Gehör für diese vollständig auf, Rot liess dagegen beide deutlicher erscheinen, während bei Gelb und Violett nur der eine Schlag verschwand.

b) Q u a litatives Hö ren. Farbenempfindungén bedingen nicht selten auch eine subjective Änderung der Tonhöhe, zumeist nur um einige Schwebungen, eine Erscheinung, "die sich deshalb meistens nur musikalischen Personen auffällig zu erkennen gibt. Sie tritt entweder gleichzeitig mit einer quantitativen Änderung der Tonempfindung oder äber obne eine solche ein. Gewöhnlich ändert sich die Tonhöhe für tiefe und hohe Töne in gleichem Sinne; also jene Farbenempfindung, die einen tiefen Ton subjectiv erhöht oder vertieft, ergibt dieselbe Veränderung auch bei einem hohen Ton, oder aber eine Tonänderung betrifft nur die tiefen oder nur die 
hohen Töne. So erfolgte bei einer Versuchsperson durch alle Farben die subjective Erhöhung eines tiefen Tones, wogegen bohe Töne unverändert blieben. In einem Falie bewirkte Grün die Erhöhung eines tiefen Tones und keine Änderung eines hohen Tones, wogegen Gelb und Blau auf tiefe Töne keinen Einfuss nahmen, dagegen hohe Töne subjectiv erhöhten. In vereinzelten Fällen besteht für tiefe und hohe Töne ein entgegengesetztes Verhalten; beispielsweise erhöhte Violett in zwei Fällen die tiefen Töne und vertiefte die hohen. Ausser diesen auf Vertiefung oder Erhöhung des einzelnen Tones beruhenden Änderungen im qualitativen Hören finden sich noch solchevor, die durch das stärkere Hervortreten einzelner Töne oder das. Zurücktreten anderer, bei gleichzeitiger Einwirkung mehrerer Töne, wie z. B. in einem Geräusche bedingt sind. So gaben mir mehrere. Versuchspersonen bei gewissen Farbenempfindungen eine Tonerhöhung in Geräusche des Uhrtickens an, die nicht durch eine tatsächliche. Erhöhung der Töne im Geräusche, sondern durch ein Zurücktreten. der tiefen Töne vorgetäuscht wurde.

Unter meinen Versuchsfällen wären die von dem Compositeur Herrn Professor Grädener und dem Violinvirtuosen Herrn. Winkler angestellten Beobachtungen hervorzuheben. Bei Herrn. Grädener erfolgt regelmässig durch Grün und Blau eine Vertiefung, durch Rot und Gelb eine Erhöhung um einige Schwebungen. sowohl tiefer als hoher Stimmgabeltöne, - gleichgiltig, ob die betreffende farbige Glastafel nur einem Auge oder beiden Augen vorgehalten wird. Bei dem Versuche mit einem Auge tritt die erwähnte Tonveränderung schwächer auf als bei binokulärer Farbeneinwirkung, betrifft aber nicht nur das Ohr der entsprechenden, sondern auch das der anderen Seite. - Bei Herrn Winkler findet in dem Augenblicke, wo eine farbige Glastafel beiden Augen vorgehalten wird, eine Erhöhung eines Stimmgabeltones statt, am stärksten bei Rot und Grün, weniger auffällig bei Gelb und Blau. Wirkt die betreffende Farbentafel durch mehrere Sekunden ein, so. zeigt sich, dass dabei keine wirkliche Tonerhöhung besteht, sonlern diese nur durch Veränderung der Klangfarbe, und zwar durch eine. höhere Klangfärbung, vorgetäuscht wird. Bei kurzdauernder Farbeneinwirkung erregt diese Erhöhung der Klangfarbe den Eindruck einer Tonerhöhung, die im Augenblicke des Entfalles der Farbeneinwirkung verschwindet.

c) Localisation der Tonempfindung. Wie ich bereits 
in diesem Archive ${ }^{1}$ ) erwähnte, wird die einzelne Tonempfindung an einer von der Tonhöhe abhängigen Stelle des Ohres oder des Kopfes localisiert, und zwar liegt diese beim monotischen Hören im Ohr und dessen Umgebung, beim diotischen Hören in den Ohren oder in vielen Fällen im Kopfe. Die einem bestimmten Tone zukommende subjective Localisationsstelle zeigt sonst bei den verschiedenen Versuchen im einzelnen Falle zumeist dieselbe Lage; bei Einwirkung der verschiedenen Farben auf die Augen kann jedoch eine Änderung dieser Localisationsstelle eintreten. In mehreren Fällen erfolgte bei Farbeneinwirkungen eine Verschiebung der im $\mathrm{Obr}$ befindlichen Localempfindung nach hinten gegen das Hinterhaupt, in einem Falle nach aussen gegen den Ohreingang. Eine derartige Verschiebung kann durch alle Farbenempfindungen in gleicher Weise stattfinden, oder sie besteht nur bei gewissen Farben. So zeigten in einem Falle ausschliesslich Blau und Violett eine Verschiebung nach hinten, wogegen bei den übrigen Farben keine Localisationsänderung eintrat. In einem Falle fand eine Veränderung des subjectiven Hörfeldes durch alle Farben, bei einer Versuchsperson durch alle Farben, ausser Gelb, statt. Dieselbe Farbenempfindung wirkt auf die Verschiebung der Localisationsstellen tiefer und hoher Töne nicht immer in gleicher Weise ein, so zwar, dass eine bestimmte Farbenempfindung zuweilen nur die Localisation eines tiefen Tones, aber nicht auch die eines hohen Tones verändert und umgekehrt. In einem Falle bewirkten Rot, Grün und Violett die Verschiebung der in der Tiefe des Gehörgangs gelegenen Localisationsstelle eines tiefen Tones nach aussen gegen den Ohreingang, indes sich Gelb und Blau ohne Einfluss erwiesen; die Localisationsstelle des hohen Tones wurde in diesem Falle durch Rot, Gelb und Violett ebenfalls nach aussen versehoben und blieb durch Grün und Blau unverändert. Es verhielten sich also in diesem Falle Rot und Violett für die Veränderung der Localisation des tiefen und des hohen Tones in übereinstimmender Weise, Blau war indifferent, Grün wirkte nur auf die Localisation des tiefen, Gelb auf die des hohen Tones ein.

Viel auffälliger als beim monotischen Hören zeigte sich in meinen Fällen eine Veränderung der in beide Ohren oder in den Kopf verlegten Localisationsstellen beim diotischen Hören. Durch

1) Über das subjective Hörfeld. Bd. 24. 1881. - Über die Localisation der Tonempfindungen. Bd. 101. 1904. 
die verschiedenen Farbeneinwirkungen verschiebt sich das im Kopf gelagerte Hörfeld gegen das eine oder andere $\mathrm{Ohr}$ oder in der Richtung von vorn nach hinten, also gegen die Stirn oder das Hinterhaupt; bald wieder treten neue Localisationsstellen hinzu, oder es: verschwinden früher vorhandene. Zuweilen betreffen die Veränderungen nur das eine $\mathrm{Ohr}$ und erstrecken sich nicht auch auf das andere. Als: Beispiele wären folgende anzuführen: Eine Versuchsperson vernabm den diotisch zugeleiteten Stimmgabelton an beiden Ohren in der Tiefe des Gehörganges; Grün, Gelb und Violett: erwiesen sich indifferent, Rot bewirkte nur am rechten Ohre eine Verschiebung der Localisationsstelle gegen die rechte Hinterhaupthälfte, bei Blau rückte die Localisationsstelle im rechten Ohre gegen die rechte Schläfe vor. Es ergab sich demnach in diesem Falle durch Rot eine Verschiebung nach hinten, durch Blau nach vorne. Das linke Obr, das gleich dem rechten eine normale Hörschärfe aufwies, zeigte durch die verschiedenen Farben keine Veränderung seiner Localisationsstelle. - In einem anderen Falle, wo sich das subjective Hörfeld des diotisch zugeleiteten Tones in der Kopfmitte befand, trat bei Einwirkung von Grün, Gelb und Violett eine Verschiebung des Hörfeldes nach rechts ein; bei Rot und Blau blieb das Hörfeld unveı ändeit. - Ein College mit beiderseits normalem Gehör verlegte bei Zuleitung eines Stimmgabeltones zu beiden Obren das subjective Hörfeld in die Kopfmitte und hatte in den Ohren selbst keine Tonwahrnehmung. Im Augenblicke der Einwirkung der verschiedenen Farben erfolgte eine Abschwächung des Toneindruckes in der Kopfmitte, bei gleichzeitigen Auftreten des Tones: in beiden Ohren. Mit Entfall des den beiden Augen vorgesetzten farbigen Glases verschwand regelmässig die Tonwahrnehmung in den Ohren, uná gleichzeitig damit trat eine Verstärkung des Toneindruckes in der Kopfmitte auf.

In mehreren Fällen erfolgten während der Farbeneinwirkung; mit oder ohne Lageänderung des subjectiven Hörfeldes, noch Änderungen in der Stärke und Höhe der Tonempfindungen. In einem Falle, wo der diotisch zugeleitete Stimmgabelton nur im rechten Ohre gehört wurde, zeigte sich regelmässig durch Rot eine bedeutende Abschwächung der Tonwahrnehmung bis zu dem vollständigen Verschwinden, durch Gelb eine Tonverstärkung und eine Erhöhung des Tones ohne Verschiebung des subjectiven Hörfeldes, durch Grün eine Tonverstärkung bei gleichzeitiger Ausbreitung des subjectiven Hör- 
feldes vom rechten Ohr bis zur Kopfmitte, wobei mit Entfall von Grün eine Abschwächung des Toneindruckes erfolgte mit gleiehzeitigem Zurückwandern des subjectiven Hörfeldes aus der rechten Kopfhälfte in das rechte Ohr; Blau und Violett erregten eine Verstärkung des Toneindruckes im rechten Ohre. - Bei einer Versuchsperson,; die den diotisch zugeleiteten Ton in die rechte Schläfe localisierte; wurde dieser durch Gelb verstärkt, durch Grün und Blau dumpfer, durch Violett bis zum Verschwinden abgeschwächt; Rot bewirkte eine "Verschiebung des subjectiven Hörfeldes mach oben, ohne Änderung des Tones. - In einem anderen Falle, wo sich das subjective Hörfeld ebenfalls in der rechten Schläfengegend befand, veranlassten Rot und Violett eine verstärkte Tonempfindung, bei Gelb erhöhte sich der Ton, bei Grün verbreiterte sich das subjective Hörfeld bis zur Kopfmitte, womit gleịchzeitig eine Tonverstärkung: erfolgte, wogegen das subjective Hörfeld bei Blau etwas nach aufwärts gegen die Kopfinitte rückte und dabei der Ton dumpfer erschien.

Wie ich bereits a. a. $0 .{ }^{1}$ ) erwähnte, können bei gleichzeitigem Anblasen beider Gehörgänge (durch einen dreischenkligen Schlauch) die diotissch einwirkenden Luftstösse die Erscheinung eines Donnerrollens hervorrufen, wobei die Schallempfindung in beide Ohren, in den Kopf oder ausserbalb des Kopfes verlegt wird. In einem Falle, wo bei gleichzeitigem Anblasen beider Gehörgänge im linken $\mathrm{Qhr}$ ein schwacher Schalleindruek, im rechten Ohre ein starker Schalleindruck und gleichzeitig ein subjectives Hörfeld entstanden, das sich vom rechten Ohr bis zur Stirnmitte erstreckte, ergab Rot eine beträchtliche Schallverstärkung in beiden Ohren, wobei auch vom linken (und nicht, wie sonst, , nur vom rechten) Ohre aus ein subjectives Hörfeld bis zur Stirnmitte reichte; bei Entfall von Rot ging dieses Hörfeld, gleichzeitig mit der gesteigerten Schallempfindung an beiden Ohren, wieder zurück. Gelb schwächte den Toneindruck an beiden Ohren und brachte das subjective Hörfeld in der rechten Kopfhälfte zum Schwinden. Grün und Violett ergaben eine bedeutende Abnahme der Hörempfindung, so dass der diotisch zugeleitete Schall nur mit dem rechten Ohre schwach gehört wurde. Bei Blau verșchwand der. Schalleindruck im linken Ohre und zeigte sich nunmehr in geringem Grade im rechten Ohr und in der rechten Kopfhälfte.

1) Pflǜger's. Archiv Bd. 24, 1881 . 
d) Subjective Gehörs empfindunge n können durch Farbeneinwirkungen eine Änderung ihrer Stärke, Klangfarbe und Localisation erleiden. Bei gleichzeitig vorhandenen verschiedenen subjectiven Gehörsempfindungen kann die einzelne Farbenempfindung: einen ausschliesslichen oder vorzugsweisen Einfluss auf die eine Art der subjectiven Gehörsempfindung nehmen; so ist auch bei subjectiver Gehörsempfindung an beiden Ohren der Einfluss auf das rechte und linke Ohr nicht immer übereinstimmend und gibt sich manchmal nur auf einem Ohre zu erkennen. Zumeist wirkt eine binokuläre Farbenempfindung stärker ein als eine monokuläre, oder die Veränderungen der subjectiven Geräusche treten überhaupt nur bei einer binokulären Farbeneinwirkung auf. Bei einer monokulären Farbeneinwirkung wieder zeigen sich mitunter vom rechten Auge aus andere Veränderungen in der subjectiven Gehörsempfindung als vom linken Auge aus. Der Einfluss auf die subjective Gehörsempfindung gibt sich gewöhnlich unmittelbar nach Beginn der Farbeneinwirkung zu erkennen und schwindet gewöhnlich gleichzeitjg mit dieser, zuweilen aber erst nach einigen Secunden, besonders wenn die Farbeneinwirkung durch längere Zeit stattgefunden hat. In einzelnen Fällen von Abschwächung der subjectiven Gehörsempfindung erschienen diese nach einer $5-15$ Minuten andauernden Farbeneinwirkung durch eine bis mehrere Stunden vollständig geschwunden oder bedeutend abgeschwächt. Als Beispiel eines verzögerten Einflusses der Farbenempfindung auf die Stärke des subjectiven Ohrgeräusches wäre ein Fall anzufübren, wo das Ohrensausen durch Violett erst nach 10-15 Secunden eine Abschwächung erfuhr und sich nach Eutfall von Violett erst 20 Secunden später in der gewöhnlichen Stärke zeigte.

Unter 20 Fällen erfolgte eine Verstärkung der subjectiven Gehörs. empfindungen durch Rot 8 mal, Gelb 3-, Grün 5-, Blau 0-, Violett $1 \mathrm{mal}$, eine Abschwächung durch Rot 8-, Gelb 10-, Grün 11-, Blau 15-, Violett $12 \mathrm{mal}$; ohne Einwirkung blieben Rot 4-, Gelb 6-, Grün 4-, Blau 5-, Violett 7 mal. In einem Falle steigerte Gelb die subjectiven Gebörsempfindungen auf dem einen $\mathrm{Ohr}$ und verminderte sie am anderen. - In einem anderen Falle verstärkten sich die subjectiven Gehörsempfindungen unter dem Einflusse von Grün und nahmen dabei einen metallischen Charakter an, der sich mit Entfall von Grun wieder verlor; Rot steigerte in diesem Falle die subjective Gehörsempfindung, ohne deren Klangfarbe zu ändern. - Bei einer 
Versuchsperson ging ein taktmässiges Klopfen im Ohr durch Rot in ein Sausen über, in einem anderen Falle ein subjectives Wasserrauschen durch Violett in ein Zischen. - In einem Falle von gleichzeitigem Klingen und Sausen wirkten Rot und Grün beruhigend nur auf das Klingen, Gelb, Blau und Violett nur auf das Sausen ein. Ein Fall von Klingen und Sausen in beiden Ohren, besonders im rechten Obr, ergab durch Rot eine Beruhigung der Geräusche an beiden Ohren, durch Grün und Blau ein Verschwinden des Klingens in beiden Ohren und eine Abschwächung des Sausens (besonders durch Blau); bei Gelb verschwand das Sausen in beiden Ohren, das Klingen am linken Ohre, wogegen im rechten Ohr ein dumpfes Klingen bestand; Violett erwies sich für das linke Ohr indifferent, schwächte dagegen am rechten Ohre beide Geräusche ab. - Ein Fall von Seidenrauschen und dem Geräusche eines Wasserfalles an beiden Olıren zeigte bei Rot ein verstärktes Wasserrausehen am linken Ohre, bei Gelb und Grün ein dumpfes Wassergeräusch nur am linken Ohre; bei Blau hörten beide subjectiven Geräusche an rechten Obre auf, wogegen am linken Ohre das Seidenrauschen unverändert anbielt, das Wasserrauschen dagegen verstärkt erschien; Violett schwächte beide Geräusche am rechten und linken Ohre wesentlich ab.

Betreffs der Farbeneinwirkungen auf beide Augen oder nur auf ein Auge ist der früher erwähnte Fall mit Sieden und Klingen an beilen Ohren (besonders rechterseits) beachtenswert. In diesem ergab Rot binokulär eine Abschwächung beider Geräusche an beiden Ohren, monokulär nur vom linken Auge aus. Bei Gelb versehwand bei binokulärer Einwirkung das Sieden an beiden Ohren, das Klingen nur am linken Ohre, wogegen es am rechten dumpfer wurde; bei Einwirkung von Gelb nur auf das rechte Auge verschwand das subjective Geräusch am entgegengesetzten linken Ohre und blieb am rechten Ohre unverändert, wogegen vom linken Auge aus am linken Ohre ein Verstummen der Geräusche und am rechten Ohre deren Abschwächung stattfand. Grün zeigte binokulär oder monokulär eine Abschwächung beider Geräusche an beiden Ohren. Bei Blau verschwand bei binokulärer Einwirkung das Klingen an beiden Ohren, das Sieden wurde bedeutend abgeschwächt; vom rechten Auge aus sistierte Blau vom linken Ohre beide Geräusche und schwächte am rechten Ohre das Klingen, nicht aber das Sieden ab; vom linken Ohre aus verstummte in gleicher Weise am entgegengesetzten rechten Ohre das Klingen, während sich das Sieden steigerte. Violett rief 
bei binokulärer Einwirkung nur an rechten Ohre eine Abschwächunğ beider Geräusche hervor, so auch vom rechten Auge aus, während vom linken Auge aus eine Abschwächung des Siedens, nicht aber auch des Klingens erfolgte.

\section{Einfluss der Farbenempfindungen anf Scheinbewegungen und Störungen des Gleichgewichts.}

Wie ich bereits a. a. $0^{1}{ }^{1}$ ) mitgeteilt habe, vermögen die verschiedenen Farbenempfindungèn einen Einfluss auf Scheinbewegungen und Störungen des Gleichgewichts zu nehmen. Es können närlich die dem Auge vorgelegten horizontalen und vertikalen Linien durch die verschiedenen Farbenempfindungen scheinbar abgelenkt werden; wobei sich die Ablenkung, je naeh der auf das Auge einwirkenden Farbe, verschieden gross zeigt und wieder die durch akustische Einwirkungen hervorgerufenen Scheinablenkungen zuweilen durch die verschiedenen Farben teilweise oder vollständig corrigiert, manchmal dagegen weiter gesteigert werden. Auch die durch akustische Reize hervorgerufenen Störungen des Gleichgewichtes und Sturzbeweghingen können durch die yerschiedenen Farbenempfindungen wesentliche Veränderungen erfahren,: wobei jeder Farbe ein eigentümlicher Einfluss zukommen kann. Dieser tritt bald in einer Änderung der Richtung einer Gleichgewichtsstörung, bald in deren Veränderung, Hemmung oder aber Steigerung hervor. In gleicher Weise zeigen sich manchmal auch spontan auftretende Gleichgewichtsstörungen durch Farben béeinflussbàr. In einem Fälle von heftigem Schwindel, wobei dér Körper beim Stehen mit geschlossenen Füssen. stetige Schwankungen nach vorn und hinten aufwies, erfolgte durch das Vorbảlten eines grüneñ Glases vor den Augen eine bedeutende Beruhigung im Schwanken; durch Rot; Gelb, Blau und Violett trat: dagegen ein Schwanken nach vorne auf, als ob der Körper von der betreffenden Glastafel magnetisch angezogen : wäre. Bei längerer Einwirkung einer der letztgenannten Farben entstand eine Sturzbewegung nach vorne. Unmittelbar nach Entfall der Farbeneinwirkung stellten sich wieder die früheren Schwankungen nach vorne und hinten ein.

1) Zeitschr. f. Ohrenheilk. Bd. 31 S. $269-294 . \quad 1897$ - - Pflüg er's Archiv Bd. 94 S. $385-388$. 
Über den Einfluss der Farbenempfindungen auf die Sinnesfunctionen. 103

Farbenempindungen vermögen ferner Scheinbilder zu beeinflussen. Hierher gehören die durch Farbenempfindungen erfolgenden Veränderungen der auf einen äusseren Reiz hin neben schwarzen Scheiben, Punkten oder Linien auftretenden Scheinbilder ${ }^{1}$ ), ferner die je nach der Farbe verschiedenartige Beeinflussung vorhandener Doppelbilder ${ }^{2}$ ). Über den Einfluss der Farben objectiver Linien. auf das Verhalten von Scheinlinien sowie über die Abhängigkeit der, Scheinbilder von der Farbe des Gesichtsfeldes habe ich bereits in diesem Archive berichtet ${ }^{3}$ ).

\section{Einfluss der Farbenempfindungen auf den Geschmacksinn.}

Die Prüfungen des Finflusses der Farbenempfindungen auf den Geschmacksinn fanden in der Weise statt, dass die Ränder der vorderen Zungenhälfte bis zur Zungenspitze von der aus dem Munde herausgestreckten Zunge mit der zur Untersuchung benutzten $\mathrm{Ge}$ schmacksflüssigkeit oder mit feinem Pulver des Geschmacksmittels bestrichen und bei herausgestreckt bleibender Zunge die einzelnen Farbentafeln den Augen zu wiederholten Malen vorgehalten und nach einigen Secunden wieder entfernt wurden. Bei vielen Personen wurden derartige Versuche ofters, dabei auch an verschiedenen Tagen angestellt. Die durch Farbenempfindungen herbeigeführten Veränderungen in der Stärke des Geschmackes zeigen sich bei derartig vorgenommenen Versuchen zumeist unmittelbar nach erfolgter Farbeneinwirkung und gehen nach deren Entfall gewöhnlich rasch zurück. Unter 15 Personen, bei denen die Geschmacksempfindung von Süss durch Farbenein wirkungen eine Änderung erfuhr, ergaben die einzelnen Farben folgendes: Rot bewirkte eine Geschmackssteigerung in fünf, eine Herabsetzung in vier, keine Geschmacksänderung in sechs Fällen; Gelb eine Steigerung in sieben, eine Herabsetzung in vier, keine Änderung in vier Fällen; Grün eine Steigerung in sechs, eine Herabsetzung in fünf, keine Änderung in vier Fällen; Blau eine Steigerung in sịeben, eine Herabsetzung in vier, keine Anderung in vier Fällen; Violett eine Steigerung in drei, eine Herabsetzung in sechs, keine Änderung in sechs Fällen. Eine Steigerung der süssen Geschmacks: empfindung bewirkte demnach in diesen fünfzebn Fällen Rot in fünf,

1) Siehe Pflüger's Archiv Bd. 94. S. 389.

2) Siehe Zeitschr. f. Ohrenheilk. Bd. 31 S. 291 e.

3) Bd. 94 S. $390-395$. 
Gelb in sieben, Grün in sechs, Blau in sieben, Violett in drei Fällen; eine Verminderung Rot in vier, Gelb in vier, Grün in fün, Blau in vier, Violett in sechs Fällen; keine Änderung ergaben Rot in sechs, Gelb in vier, Grün in vier, Blau in vier, Violett in sechs Fällen.

Eine vergleichsweise Prüfung mit verschiedenen Geschmacksarten ergibt zum grossen Teile eine übereinstimmende Einwirkung der einzelnen Farben auf Süss, Salzig, Sauer und Bitter, so dass die Farbenempfindung, bei der z. B. eine Steigerung der Geschmacksempfindung von Süss stattfindet, auch eine solche für Salzig, Sauer oder Bitter aufweist. Doch können sich dabei auch Verschiedenbeiten in der Art der Einwirkung zeigen, so zwar, dass dieselbe Farbe bei der einen Geschmacksart eine Steigerung, bei den anderen eine Herabsetzung der Empfindung bewirkt oder die Geschmacksempfindung unverändert lässt. Beispielsweise fand bei einer Versuchsperson durch Rot eine Geschmackssteigerung für Salzig, Sauer und Bitter statt, wogegen Rot für Süss indifferent blieb; Gelb verminderte die Geschmacksempfindung von Süss, Salzig und Sauer und steigerte die von Bitter; durch Grün fand eine beträchtliche Steigerung der Geschmacksempfindung von Süss statt, eine geringe von Salzig und Sauer und keine Änderung von Bitter; Blau verändert den süssen Geschmack, steigerte den salzigen und bitteren und liess den saueren unverändert; Violett steigerte den süssen und bitteren Geschmack und vermindert den von Salzig und Sauer.

Versuche mit Einwirkung der Farben nur auf ein Auge ergaben bäufig eine Geschmacksänderung nur auf der gleichseitigen Zungenhälfte. Dabei erscheint bei sonst gleich starker Geschmacksempfindung der beiden Zungenhälften die Einwirkung vom rechten und linken Auge aus nicht selten ungleich, indem beispielsweise dieselbe Farbe bei ihrer Einwirkung auf das rechte Auge ein Auslöschen der Geschmacksempfindung am rechten Zungenrande ergeben kann, indes vom linken Auge aus nur eine Schwächung des Geschmacks am linken Zungenrand erfolgt.

Vergleichsweise Geschmacksprüfungen bei monokulärer und binokulärer Farbeneinwirkung zeigen in manchen Fällen auffallende Verschiedenheiten betreffs der Stärke der geänderten Geschmacksempfindungen und der Stelle ihres Auftretens. So trat bei einer Versuchsperson, wenn diese mit dem linken Auge durch ein gelbes Glas sah, am linken Zungenrande eine Geschmackssteigerung auf, wogegen sich voin rechten Auge aus keine Änderung der Geschmacks- 
Über den Einfluss der Farbenempfindungen auf die Sinnesfunctionen. 105

stärke zu erkennen gab; bei binokulärer Einwirkung von Gelb erfolgte jedoch an beiden Zungenrändern eine Geschmackssteigerung, nur erschien diese links stärker als rechts.

Die Schwächung der Geschmacksempfindungen durch Farbeneinwirkung kann bis zum vollständigen Auslöschen der Geschmacksempfindung führen, entweder an allen von der Geschmackssubstanz betroffenen Zungenstellen oder nur an einem Teile dieser; anderseits wieder treten bei einer Steigerung der Geschmacksempfindungen mitunter neue Geschmacksstellen hinzu, d. h. infolge der Steigerung des Geschmackes werden vorher nicht wahrgenommene Geschmacksempfindungen über die Bewusstseinsschwelle gehoben. Man hat demnach bei einer durch Farbeneinwirkung erfolgenden Veränderung der Geschmacksstärke nicht allein auf deren Verbalten an den geschmacksempfindenden Zungenstellen vor dem Farbenversuche zu achten, sondern auch auf eine etwaige Änderung betreffs der Ausdehnung des Gesehmacksgebietes. So erfolgte in einigen Fällen von Geschmackssteigerung gleichzeitig eine Vergrösserung der Geschmacksfläche, indem sich die Geschmacksempfindung einerseits über einen breiteren Streifen des Zungenrandes erstreckte wie sonst und andererseits auch gegen die hintere Zungenpartie ausdehnte. Mit Entfall der Farbeneinwirkung zog sich wieder die Geschmacksempfindung auf den Zungenrand selbst und auf die vordere Zungenpartie zurück. Bei mehreren Versuchspersonen, bei denen durch bestimmte Farben eine Geschmacksverminderung erfolgte, gab sich gleichzeitig mit dieser eine Einschränkung des Geschmacksgebietes zu erkennen. Eine solche Einschränkung betrifft zumeist die Partien seitlich von. den Zungenrändern und ferner die hintere Zungenhälfte. In einem Falle jedoch schwand bei den verschiedenen Farbeneinwirkungen der Salzgeschmack an der Zungenspitze und wurde nur in der mittleren. Zungenpartie am Zungenrande wahrgenommen; bei Süss, Sauer und Bitter gab sich an der Zungenspitze und an den Zungenrändern der vorderen Zungenhälfte nur eine Änderung der Geschmacksstärke ohne solehe des Geschmacksgebietes zu erkennen. Bei einer Versuchsperson erfolgt durch eine bestimmte Farbeneinwirkung eine Verschmälerung des geschmacksempfindenden Saumes am Zungenrande, wobei aber an der übrigbleibenden linienförmigen Geschmacksstelle des Zungenrandes der Gesehmack besonders deutlich hervortrat. Die betreffende Person versicherte, dass diese Geschmackssteigerung tatsächlich als solche bestünde und nicht etwa auf einer 
Art Contrasterscheinung infolge Abnahme :des Geschmackes an den benachbarten Zungenpartien beruhe.

Mitunter gibt sich unter dem Einflusse einer bestimmten Farbenempfindung eine qualitative Änderung des Geschmackes zu erkennen. Bei einzelnen Versuchspersonen erhielt der Geschmack von Zucker unter der Einwirkung von Grün einẹ säuerlichen Beigeschmack, der sich mit Entfall von Grün wieder verlor. Bei einem Collegen bewirkten Rot und Grün regelmässig, Blau und Violett nicht an allen Versuchstagen eine Änderung des Zuckergeschmackes in eine bittere Geschmacksempfindung, die so lange anhielt, als die betreffenden Farben einwirkten; nach deren Entfall trat an den Zungenrändern wieder der süsse Geschmack auf. Bei Application von Chinin an die Zungenränder wurde die bittere Geschinacksempfindung durch Rot und Grün verstärkt. Gelb verhielt sich indifferent. - In einem Falle entstand durch Gelb eine Änderung des -süssen Geschmackes in einen bitteren, durch Rot, Grün, Blau in eine saure Geschmacksempfindung, welche Geschmacksumstimmung jedesmal im Augenblick der Farbeneinwirkung auftrat und mit deren Entfall wieder zur süssen Geschmacksempfindung zurückging.

Eine Beeinflussung des Geschmacks durch Farbenempfindungen betrifft auch den galvanisch e rregten Geschmack. In fünf Fällen, wo die Anode der Zunge anlag, erfuhr der laugenartige Anodengeschmack durch die verschiedenen Farben folgende Veränderung: Eine Steigerung fand durch Rot, Gelb und Grün je zweimal statt, durch Blau und Violett je einmal; eine Verminderung ergaben Rot und Blau je dreimal, Gelb und Grün je zweimal, Violett einmal; indifferent blieb der Anodengeschmack bei Violett dreimal, bei Gelb, Grün und Blau je einmal.

Ausser dem erwähnten Einflusse auf die Stärke der Geschmacksempfindungen vermögen Farbeneinwirkungen auf den Tast- und Temperatursinnder Zunge einzuwirken und die Speichelsecretion anzuregen. So tritt mitunter bei Einwirkung einer bestimmten Farbe ein Kitzeln, Kratzen oder Brennen am Zungenrande auf, ein andermal wieder eine kühle Empfindung, als ob der Zunge ein kalter Gegenstand angelegt würde. Diese Erscheinungen zeigen sich entweder gleichzeitig mit einer Änderung der Geschmacks;stärke oder ohne: solche. Zuweilen vermischen sich die Geschmácksempfindungen mit den neu auftretenden Tastempfindungen, wodurch eine Änderung. dès Geschmackes vorgetäuscht werden kann. So 
wurde in einzelnen Fällen das mit der Farbenieinwirkung zu dem süssen Geschmacke hinzutretende Brennen am Zungenrande auf eine neu aufgetretene saure Geschmacksempfindung bezogen. Noch leichter tritt eine solche Täuschung bei einem sauren Geschmacke auf, : wo die hinzukommende prickelnde oder stechende Empfindung leicht für eine Verschärfung des sauren Geschmackes gehalten wird.

Bemerkenswerter Weise zeigte sich in der Mehrzahl meinier Versuchspersonen die dureh Farbeneinwirkung ausgelöste sensitive :Empfindung nur bei gleichzeitig vorhandenen Géschmacksempfindungen in der Weise, dass durch die betreffende Farbeneinwirkung nur an den Zungenrändern, wo der Geschmack erregt worden war, eine sensitive Empfindung hinzutrat, wogegen dieselben Zungenpartien ohne vorhandenen Geschmacksreiz durch Farbeneinwirkungen keinerlei sensitive Empfindungen aufwiesen. Vielleicht beruht diese Erscheinung darauf, dass durch das Geschmacksmittel ein gewisser Reiz auf die sensitiven Nerven ausgeübt wird, der als solcher nicht auffällig hervortritt, wogegen bei einer weiteren, wenn auch unbedeutenden Erregung dieser Nerven, durch die Farbèneinwirkung nunmehr eine merkliche sensitive Empfindung , erfolgt. Eine solche kann sich auch in Fällen zeigen, wo die Geschmacksempfindung dürch die Farbeneinwirkung keine Änderung erfährt. So erwiesen sich in einem Falle sämmtliche Farben auf die Geschmacksstärke indifferent; dagegen erregte Rot regelmässig ein Kitzeln am rechten, mit Zucker bestreuten Zungenrande, am linkęn ein Kratzen, wobei am linken Zungenrande gleichzeitig, eine kühle Empfindung auftrat; dabei war keine Änderung im Geschmacke bemerkbar. In einem Falle, wo der Zungenrand beiderseits mit feinem Zuckerpulver bestrichen worden war, entstand, bei Einwirkung yonn Rot oder Violett auf das rechte Auge, am rechten Zungenrande die Empfindung des Küblen, wogegen vom linkè Auge aus keine Gefühlsempfindung ausgelöst wurde; bei Einwirkung von Rot oder Violett, gleichzeitig auf beide Augen, entstand an der Zunge die Empfindung des Küblen; und zwar bei Rot an beiden Zuningenrändern, bei Violett nur an der Zungenspitze.

\section{Einfliuss dér Farbenempfindungen auf den Geruch.}

Eine Untersuchung über den Einfluss der Farbenempfindungen auf den Geruch ergab in zehn Fällen Nachstehendes: Erster Fall: 
Alle Farben vermindern die Geruchsempfindungen. Zweiter Fall: Rot steigert den Geruch, die übrigen Farben setzen ihn herab. Dritter Fall: Rot und Blau vermindern den Geruch, Gelb, Grün und Violett verhalten sich indifferent. Vierter Fall: Rot schwächt den Geruch, die übrigen Farben steigern ihn. Fünfter Fall: Alle Farben steigern die Geruchsempfindung. Sechster Fall: Rot und Gelb steigern, Blau und Violett schwächen den Geruch, Grün ist indifferent. Siebenter Fall: Rot steigert, Gelb, Blau und Violett vermindern den Geruch, Grün bleibt indifferent. Achter Fall: Alle Farben steigern den Geruch. Neunter Fall: Rot, Gelb, Violett vermindern, Blau steigert den Geruch, Grün verbält sich infifferent. Zehnter Fall: Rot steigert den Geruch, die übrigen Farben verhalten sich indifferent.

Unter diesen zehn Fällen erfolgte demnach durch Rot sechsmal eine Steigerung des Geruches, viermal eine Verminderung; durch Gelb viermal eine Steigerung, viermal eine Verminderung, zweimal keine Änderung; durch Grün dreimal eine Steigerung, zweimal eine Verminderung, fünfmal keine Änderung; durch Blau viermal eine Steigerung, fünfmal eine Verminderung, einmal keine Änderung; durch Violett dreimal eine Steigerung, fünfmal eine Verminderung, zweimal keine Änderung. In zwei dieser Fälle fand durch alle Farben eine Steigerung, in einem Falle eine Verminderung der Geruchsempfindungen statt. In den beiden Fällen von Geruchsstejgerung erwies sich Grün von besonders erregender Wirkung; die geringste Steigerung ergaben Blan und Violett. In dem Falle von Geruchsverminderung durch alle Farben trat eine geringe Abschwächung des Geruches durch Rot und Blau ein, eine bedeutende, bis zum Auslöschen des Geruches, durch Gelb, Grün und Violett. - In einem Falle, wo das Geruchsvermögen auf der linken Nasenseite schwächer erschien als auf der rechten, erfolgte bei einer Einwirkung von Gelb, Grin, Blau und Violett auf das linke Auge eine Steigerung des Geruches auf der linken Nasenseite, besonders durch Blau und Violett, so dass während der Einwirkung einer dieser beiden Farben die Geruchsempfindung auf beiden Nasenseiten gleich stark erschien; unmittelbar nach Entfall von Blau und Violett trat wieder die stärkere Geruchsempfindung auf der rechten Nasenseite hervor. 
Über den Einfluss der Farbenempfindungen auf die Sinnesfunctionen. 109

\section{Einfluss der Farbenempfindungen auf den Tast- und Temperatursinn.}

Der Einfluss der Farbenempfindungen auf den Tastsinn wurde in der Weise geprüft, dass während des Streichens identischer Hautstellen der rechten und linken Körperhälfte (z. B. der Stirn, Wangen oder der Hände) mittelst eines einzelnen Pinselhaares die verschiedenen Farben vor die. Augen gehalten und wieder entfernt wurden, was bei jedem Versuche wiederholt geschah. Zur Prüfung der Temperaturempfindung wurde ein in sehr kaltem oder heissem Wasser gelegener Metallhammer vor, während und nach der Farbeneinwirkung den verschiedenen Körperstellen aufgelegt. Es erwiesen sich auch hierbei die verschiedenen Farbenempfindungen von Einfluss auf eine Steigerung oder Abschwächung des Tast- und Temperatursinnes, während einzelne Farben indifferent blieben.

Unter den Veränderungen der Tastempfindungen wäre $\mathrm{zu}$ bemerken, dass sich bei einzelnen Personen die verstärkte Empfindung des Pinselhaarstreichens in einem vermehrten Kitzeln, Kratzen ode' einer stechenden Empfindung äussert, die abgeschwächte wieder in einer undeutlichen Streichempfindung, wobei der Pinselstrich entweder auf derselben Hautstelle undeutlicher gespürt wurde oder aher scheinbar auf einer breiteren Hautstelle. Die betreffenden Versuchspersonen haben im letzten Falle den Eindruck, als ob das Pinselhaar weicher und breiter würde und bei Entfall der Farbeneinwirkung wieder härter und feiner, wobei aber der feine Pinselstrich viel deutlicher empfunden wird als der breite. Mitunter sinkt die Abschwächung der Empfindung für einen feinen Pinselstrich bei gewissen Farbenein wirkungen unter die Empfindungsschwelle und erscheint wieder bei Entfall der betreffenden Farbe.

Bezüglich der Farbeneinwirkungen auf die Tast- und Temperaturempfindungen der Zunge siehe Seite 106.

Eine vergleichsweise Prüfung einer bestiminten Farbenempfindung auf die verschiedenen Sinne ergibt in vielen Fällen einen übereinstimmenden Einfluss auf alle oder auf die Mehrzabl der Sinnesempfindungen. Wenn beispielsweise Rot eine Steigerung oder Herabsetzung der Hörempfindungen veranlasst, so tritt eine entsprechende Änderung der Empfindungsstärke häufig auch für die Geschmacks-, Geruchs- und Tastempfindungen auf. Dabei erweist sich allerdings die Veränderung in der Empfindungsstärke 
bei dem einzelnen Sinne sehr verschieden, indem.z. B. Rot eine bedeutende Steigerung des Geschmackes, aber nur eine geringe des Geruches hervorrufen kann. Zuweilen macht sich ein solcher Einfluss nur auf eine bestimmte Sinnesempfindung geltend, während sich diẹselbe Farbe den anderen Sinnesempfindungen gegenüber indifferent verhält, ja, sogar ein entgegengesetztes Verhalten aufweisen kann; so steigerte Rot in einem Falle die Hör- und Geschmacksempfindungen und verminderte die Geruchs- und die Tastempfindungen.

Was die Einwirkung einer bestimmten Farbe auf die Steigerung oder Herabsetzung der Sinnesempfindungen anbelangt, so ergeben die angeführten Beobachtungen, dass keiner der Farben in dieser Beziehung ein vorzugsweiser Eiufluss zukommt. So wirkt beispielsweise Rot keineswegs besonders häufig erregend und wieder Blau herabsetzend auf die Sinnesfunctionen, sondern in vielen Fällen ergibt in ungekehrter Weise Blau eine Steigerung und Rot eine Herabsetzung der Sinnesempfindungen. Das gleiche wechselnde Verhalten betrifft die Einwirkungen von Complementärfarben auf die Sinnesfunctionen; so können z. B. Rot und Grün bald übereinstimmend, in gleicher oder ungleicher Stärke, die Sinnesempfindügen vermehren oder vermindern, bald wieder eine entgegengesetzte Wirkung äussern, beispielsweise Rot steigernd, Grün herabsetzend einwirken, oder aber nur eine der beiden Complementärfarben ergibt einen Einfluss auf die Sinnesfunetionen, während sich die andere indifferent erweist:

Bei einer Umfrage nach der Lieblingsfarbe der einzelnen Versuchsperson fand sich häufig, dass die Farbe, bei der die stärkste Erregung der Sinnesempfindungen eintritt, als die Lieblingsfarbe bezeichnet wird, während jene Farbe, die eine Herabsetzung der versthierlenen Sinnesempfindungen veranlasst, als gleichgültig oder als unangenehm angegeben wurde.

Meinen früheren Beobachtungen zufolge kann die durch eine bestimmte Gehörserregung herbeigeführte Beeinflussung von subjectiven Gesichtsempfindungen auch durch die subjective Nachempfindung der vorausgegangenen Gehörserregung veranlasst werden $\mathbf{1}$ ). Es lag daher nahe, Versuche anzustellen, ob auch eine im Nachbilde auftretende, also rein subjective Farbenempfindung,

1) Siehe: Über die Beeinflussung subjectiver Gesichtsempfindungen. Pflüger"s Archiv Bd. 94 S. 380 u. 420. 1903. 
die verschiedenen Sinne zu beeinflussenvermag. Die darüber angestellten Untersuchungen ergaben in einer Reihe von Versuchspersonen mit lebhaft farbigen Nachbildern tatsächlich eine Übereinstimmung des Einflusses der objectiven Farbeneinwirkung mit der der gleichfarbigen subjectiven Nachempfindung (der objectiven Complementärfarbe), so zwar, dass die durch eine bestimmte Farbe herbeigeführte Zu- oder Abnahme einer Sinnesempfindung sowohl bei der objectiven als auch bei der subjectiven Einwirkung dieser Farbe erfolgte. Beispielsweise rief in einem Falle Grün eine Șteigerung, Rot eine Herabsetzung der verschiedenen Sinnesempfindungen hervor; wenn dem Auge Rot vorgesetzt wurde und hierbei eine Abschwächung der Sinnesempfindungen stattfand, so zeigte sich mit dem Eintritte des Nachbildes von Rot, also bei Grün, gleichzeitig eine Steigerung der Sinnesempfindungen, die so lange anbielt, als die Nachempfindung währte. Wenn in diesem und in anderen ähnlichen Fällen bei einer nicht genügend langen Einwirkung der objectiven Farbe kein Nachbild der Complementärfarbe auftrat, fand sich dementsprechend auch keine Änderung in der Stärke der Sinnesempfindung vor, als Zeichen, dass eine solche Änderung tatsächlich von dem farbigen Nachbilde abhängig war.

In einem Falle von subjectiven Gehörsempfindungen, die durch Rot gesteigert, durch Grün abgeschwächt wurden, zeigten die Versuche Folgendes: Fine grüne Glastafel, beiden Augen vorgesetzt, verminderte die subjectiven Gehörsempfindungen an beiden Ohren; wurden die Augen geschlossen, so trat gleichzeitig mit dem Erscheinen des roten Nachbildes eine Steigerung der Ohrgeräusche auf, die mit dem Schwinden des Nachbildes wieder zurückging. In umgekehrter Weise erregte das Durchsehen durch eine rote Glastafel ein verstärktes Ohrensausen, indes gleichzeitig mit dem Auftreten des complementären Nachbildes, Grün, eine erhebliche Verminderung des Sausens erfolgte. Als bei einem dieser "Versuche kein Nachbild auftrat, zejgte sich auch nach Verschluss der Augen nur jene Änderung in der Stärke der subjectiven Gehörsempfindungen, die auf dem Entfall der vorausgegangenen Farbeneinwirkung beruhte (es erschien also das durch die vorgesetzte rote Glastafel gesteigerte Ohrgeräusch mit Verschluss der Augen auf die frühere Stärke abgeschwächt und das durch Grün abgeschwächte Geräusch nach Entfall von Grün in entsprechender Weise wieder gesteigert), ohne dass eine weitere Änderung in der Stärke der subjectiven Gehörs- 
empfindungen stattgefunden hätte, wie bei den früheren Versuchen, wo ein Auftreten von complementären Nachbildern erfolgt war. Weitere Versuche mit gleichzeitiger Einwirkung von Rot auf das eine und von Grün auf das andere Auge ergaben in einzelnen Versuchen das Nachbild Grün, womit auch eine Abschwächung der subjectiven Geräusche eintrat; zeigte sich jedoch als Nachbild Rot, so wurde damit eine Stejgerung der subjectiven Gehörsempfindungen beobachtet. Bei einem dieser Versuche erschienen im Nachbilde zwei rote Felder, zwischen denen ein schmales grünes Feld lag ${ }^{1}$ ); dabei bestand ein vermehrtes Ohrensausen, entsprechend dem Überwiegen der roten Farbe im Nachbilde. Ein andermal trat zuerst ein rotes, dann ein grünes Nachbild auf und damit zuerst eine Steigerung, dann eine Abschwächung der subjectiven Gehörsempfindungen.

Betreffs einer Änderung des Geschmacks durch subjective Farbenempfindungen wären nachfolgende Fälle anzuführen: Bei einer Versuchsperson ergab Rot, beiden Augen vorgehalten, eine bedeutende Steigerung, Grün eine Abschwächung des süssen Geschmackes; wurde Grün den Augen durch einige Zeit vorgehalten, so ging nach Verschluss der Augen die durch Grün hervorgerufene Verminderung des süssen Geschmacks wieder zurück; mit dem allmählich stärker hervortretenden Rot des Nachbildes hob sich die Geschmacksempfindung im Verhältnisse zur Stärke von Rot in dem Nachbilde; kam dieses bei den einzelnen Versuchen nur schwach zur Entwicklung, so gab sich dementsprechend nur eine geringe Geschmackssteigerung zu erkennen, und bei fehlendem Nachbilde, z. B. bei zu kurz dauernder Finwirkung der objectiven Farbe, Grün, zeigte sich überhaupt keine Geschmackssteigerung.

Bei einem Collegen erwies sich Rot für den süssen Geschmack indifferent; Grün bewirkte eine "Geschmackssteigerung. Eine rote Glastafel, den beiden Augen vorgehalten, ergab als Nachbild ein schwach rosarotes $\mathrm{H}$, innerhalb dieses zwei tief dunkelviolette Felder, von denen das eine oberhalb, das andere unterhalb des Horizontalstriches gelegen war. Nach einigen Secunden trat am oberen violetten Felde eine tief grüne Färbung hervor, bis zum vollständigen Verdrängen von Violett. Mit dem Überwiegen der grünen Farbe ent-

1) Siehe darüber meine Abhandlung: Über die Beeinflussung subjectiver Gesichtsempfindungen. Pflüger's Archiv Bd. 94 S. 437. 1903. 
Über den Einfluss der Farbenempfindungen auf die Sinnesfunctionen. 113

stand an den Zungenrändern eine Steigerung des Zuckergeschmackes, die bis zum Abklingen des grünen Nachbildes anhielt. - Bei einem zweiten solchen Versuche mit dem Nachbilde von Rot fanden sich anstatt des $\mathrm{H}$ nur zwei senkrechte rosarote Streifen vor; das innerhalb dieser gelagerte Feld zeigte anfänglich einen Wettstreit zwischen Violett und Grün, der mit einem raschen Siege von Grün endete; gleichzeitig mit diesem steigerte sich die süsse Geschmacksempfindung an der Zunge und verlor sich erst nach dem Schwinden des grünen Nachbildes.

Eine bemerkenswerte Beobachtung fand betreffs des Einflusses farbiger Nachbilder auf die Geschmacksempfindung bei dem S. 106 erwähnten Collegen statt. Dieser hatte an früheren Versuchstagen nur durch Rot und Grün an den mit Zucker bestreuten Zungenrändern eine Änderung des süssen Geschmacks in eine bittere Geschmacksempfindung bemerkt. An einem der folgenden Versuchstage erregte eine den Augen vorgehaltene rote Glastafel eine geringe Umstimmung des süssen Geschmacks in eine bittere Geschmacksempfindung. Das Nachbild von Rot ergab anfangs eine Andeutung von Grün ohne Änderung des süssen Geschnnacks; auf Grün trat in besonderer Stärke eine dunkelblaue subjective Farbenempfindung ein, womit sich gleichzeitig eine starke bittere Geschmacksempfindung an der Zunge bemerkbar machte. Dieser Einfluss des Blau im Nachbilde widersprach dem am früheren Beobachtungstage vorgefundenen indifferenten Verhalten der Einwirkung einer blauen Glastafel auf die Augen. Als jedoch dieselbe blaue Glastafel den Augen vorgehalten wurde, zeigte sich nunmehr, dass an dem betreffenden Versuchstage auch durch ein objectives Blau die erwähnte Geschmacksänderung bewirkt wurde. Wie nachträgliche Versuche ergaben, war ein solcher Einfluss von Blau und Violett an den verschiedenen Tagen ein wechselnder und mitunter nur spurweise vorhanden. Die qualitative Geschmacksänderung durch Blau war also bei dem Collegen zuerst an dem Blau des Nachbildes beobachtet worden und erst nachträglich auch bei Einwirkung des objectiven Blau.

An einem weiteren Versuchstage, wo Rot, Grün und Blau eine deutliche Änderung des Geschmackes von Süss und Bitter hervorriefen, Gelb und Violett sich indifferent verhielten,- liess ich nach einem längeren Vorhalten der grünen Farbe vor beiden Augen diese schliessen. Damit kehrte die durch Grün hervorgerufene bittere Geschmacksempfindung in den richtigen süssen (Zucker-)Geschmack 
zurúck. Einige Secunden nach Verschluss der Augen entstand ein lebhaftes dunkelviolettes Nachbild und damit eine stark bittere Geschmacksempfindung an den mit Zucker bestreuten Zungenrändern. Bemerkenswerter Weise hatte die unmittelbar vorher angestellte Prüfung mit dem objectiven Violett den Geschmack nicht beeinflusst, während das violette Nachbild, wie erwähnt, sogar einen starken bitteren Geschmack erregte. Ich stellte daher einen Controllversuch mit dem violetten Glase an; doch verhielt sich auch diesmal die objective violette Farbe indifferent. Der College bemerkte jedoch dabei, dass die objective violette Farbe nicht dem von ihm gesehenen subjectiven Violett entspreche, sondern dass dem Violett im Nacbbilde vielmehr Blau beigemischt war. Als der violetten Glastafél eine blaue aufgelegt wurde, entstand eine dem Violett des Nachbildes entsprechende Farbe: und damit auch bei deren Einwirkung auf das Auge die früher beim Nachbilde aufgetretene Geschmacksänderung von Süss in Bitter. Es hatte sich also auch bei diesem Versuche die Einwirkung einer bestimmten Farbe auf die Geschmacksempfindung zuerst an dem subjectiven Farbenbilde zu erkennen gegeben.

Wie erwähnt, trat in dem soeben angeführten Falle, bei Verschluss der Augen, so auch im dunklen Raume, gewöhnlich eine subjective Farbenempfindung von Blau oder Blau-Violett spontan auf. Der betreffende College beobachtet diese Erscheinung seit Jabren stets in gleicher Weise, u. a. auch des Morgens beim Erwachen. Bei einer Umfrage, die ich darüber an verschiedene Personen richtete, ergab sich, dass eine anscheinend nicht durch äussere Farbeneinwirkungen veranlasste, sondern spontan auftretende subjective Farbenempfindung häufig vorkommt und verschiedene Farben betrifft. Von mehreren Personen wurde mir ein tiefes Blau angegeben; andere beobachteten regelmässig Violett oder Grün, Gelb oder Rot; in einem Falle erschien nach Verschluss der Augen und nur bei vollständiger äusserer Stille ein gelbroter Kreis, der sich rasch erweiterte und durch längere Zeit als solcher bestehen blieb. Wie mir mehrere Erwachsene mitteilten, beobachten sie seit ihrer Kindheit dieselbe spontane subjective Farbenerscheinung. Im späteren Alter kann sich eine solche verlieren; so berichtete mir ein 72jähriger Mann, dass er von Kindheit an bis in das 50. Lebensjahr bei Verschluss der Augen regelmässig ein tiefes Blau beobachtete, welche Erscheinung nach dem 50. Jahre allruählich schwand. 
Eine derartig spontan auftretende subjective Farbe verhält sich in verschiedener Weise zu einem subjectiven farbigen Nachbilde einer objectiven Farbe, die auf das Auge eingewirkt hat, und zwar können beide entweder gesondert vorkommen oder aber sich miteinander mischen. Bei einem gesonderten Auftreten erscheint bald das farbige Nachbild, bald die spontane subjective Farbe als erstes; so beobachtete 'eine Person, die bei Verschluss der Augen stets ein blaues Farbenfeld sah, nach dem Durchsehen durch ein grünes Glas und nach darauffolgendem Verschlusse der Augen einimal anfänglich ein rotes Nachbild, das nach einigen Secunden einem blauen. subjectiven Farbenfelde: wich, ein andermal dagegen zuerst die bläue und erst später die complementäre rote Farbe. Zuweilen treten beide der artige subjective Farbenempfindungen, also sowohl die spontane als auch die complementäre, in einen Wettstreit miteinander, so dass durch einige Zeit bald die eine, bald die andere subjective Fárbe vorherrséht. Mitunter kommen auch beide gleichzeitig nebeneinander zur Beobachtung. Wie bemerkt, erfolgt mitunter eine Mischung beider subjectiver Farben, wobei sich manchimal der Beginn der Mischung beider Farben und hierauf wieder deren Sonderung deutlich verfolgen lässt. In dem erwähnten Falle, wo Blau als spontane subjective Farbe auftrat, zeigte sich bei einem der Versuche, nach. dem Durchsehen durch ein grünes Glas; bei verschlossenen Augen zuerst Blau; nach kurzer Zeit ging das Blau in ein Blau-Violett über, das sich rasch in Violett mit zunehmender Beimischung von Rot änderte, bis schliesslich Rot allein im subjectiven Gesichtsfelde zur Beobachtung kam: Es hatte sich also in diesem. Falle mit dem ursprünglichen subjectiven reinen Blau das später hinzutretende subjective Rot (das Nachbild von Grün) zu Violett gemischt; durch das allmähliche Zurücktreten von Blau überwog in entsprechender Weise im violetten subjectiven Gesichtsfelde Rot immer mehr, bis nach vollständjgem Schwinden von Blau Rot schliesslich allein zurückblieb. In demselben Versuchsfalle wurde ein andermal ein ähnliches Verhalten des subjectiven Nachbildes Grün (bei vorausgegangenem objectivem Rot) mit Blau beobachtet, indem Grün, als zuerst aufgetretene subjective Farbe, in Grünblau überging, auf das rasch Blaugrün und endlich ein reines Blau (als spontane subjective Farbe) folgten.

Gleich dem subjectiven farbigen Nachbilde kann auch die spontan auftretende subjective Farbe oder die Mischung beider einen Einfluss auf die Sinnesempfindungen nehmen, ähnlich der entsprechenden 
objectiven Farbe, welcher Umstand bei den betreffenden Untersuchungen berücksichtigt werden muss. In dem Falle, wo Blau eine Änderung des süssen Geschmackes in Bitter bewirkte, erfolgte dies auch bei dem spontanen subjectiven Auftreten von Blau. - Eine Versuchsperson, die durch Rot eine Veränderung des Geschmackes erlitt, beobachtete dieselbe Erscheinung bei dem subjectiven Rot, das sich bei der betreffenden Person seit mehreren Jahren nach Verschluss der Augen einzustellen pflegt. In ähnlicher Weise verhielt es sich in einem anderen Falle mit eirer Geschmackssteigerung dureh Grün.

Auch die durch Bewegungen des Augapfels hervorgerufenen subjectiven Farbenempfindungen sind im Stande, Sinnesempfindungen zu beeinflussen. Bei dem Collegen, wo Grün den süssen Geschmack in Bitter umwandelt, zeigt sich bei raschen Bulbusbewegungen in horizontaler. Richtung oder nach unten häufig ein subjectives tiefes Grün (Bewegungen der Bulbi nach oben ergeben keine subjectiven Farbenerscheinungen); mit dem Auftreten der subjectiven grünen Farbe erfolgt stets eine deutliche bittere Geschmacksempfindung an der mit Zucker bestrichenen Zungenspitze; dagegen stellt sich diese Erscheinung nicht ein, wenn die erwähnten Augenbewegungen kein subjectives grünes Feld ergeben.

Als Beispiel einer Beeinflussung dersensitiven Nerven durch eine subjective Farbenempfindung wäre ein Fall anzuführen, wo Rot an den mit Zucker bestrichenen Zungenrändern, besonders gegen die Zungenspitze, eine stechende Empfindung hervorrief; Grün erregte diese nicht. Wenn nach vorausgegangener Einwirkung von Grün dessen farbiges Nachbild Rot auftrat, so machte sich damit gleichzeitig eine prickelnde, aber nicht, wie bei Einwirkung des objectiven Rot, stechende Empfindung am vorderen Zungendrittel bemerkbar, die mit dem Abklingen des roten Nachbildes zurückging.

In einem Falle, wo Rot eine bedeutende Steigerung des Geruches herbeiführte, entstand beim subjectiven Auftreten dieser Farbe, als des farbigen Nachbildes von Grün, ebenfalls eine Geruchssteigerung.

In einzelnen Fällen, die zumeist nervös sehr erregbare Personen betrafen, schien die Bestrahlung grösserer Hautflächen des Körpers mit farbigem Lichte einen ähnlichen Einfluss a uf die verschiedenen Sinnesempfindungen zu nehmen wie die Einwirkung der Farbe auf die Augen. Es ergab sich nämlich, 
Über den Einfluss der Farbenempfindungen auf die Sinnesfunctionen. 117

dass bei einigen Versuchspersonen die durch eine bestimmte Farbeneinwirkung auf die Augen erregte Steigerung oder Herabsetzung der verschiedenen Sinuesempfindungen in einem gewöhnlich abgeschwächten Grade auch durch Bestrahlung einer grösseren Hautfläche mit der betreffenden Farbe hervorgerufen werden konnte. Selbstverständlich hatten die Versuchspersonen bei diesen Versuchen keine Kenntnis davon, welche Farbe auf die Haut einwirkte. Ich suchte aber ausserdem die Versuchsperson über den Eintritt der jedesmaligen Farbenbestrahlung irrezuführen, täuschte öfters eine Farbeneinwirkung vor, indes tatsächlich keine solche bestand, und liess ein andermal wieder die einzelne Farbe auf die Haut unbemerkt einwirken. Die hierbei nachweisbare Übereinstimmung in der Veränderung der verschiedenen Sinnesempfindungen bei der Farbeneinwirkung auf die Haut und bei der auf die Augen erscheint mir jedenfalls beachtenswert. In einem Falle, wo die Einwirkung von Grün auf die Augen sowie auf die Handflächen eine Steigerung der Sinnesempfindungen und Blau deren Herabsetzung ergab, teilte ich der Versuchsperson, deren Augen sorgfältig verschlossen worden waren, bei einem Versuche mit Geschmacksempfindungen fälschlicher Weise mit, dass die seitlichen Partien des Kopfes und Halses mit blauem Lichte bestrahlt würden, indes dies mit grünem Lichte der Fall war. Die betreffende Person, die im Sinne der früheren Versuchsergebnisse eine Abnahme der Geschmacksempfindung erwartete, beobachtete zu ihrer Überraschung eine Steigerung des Geschmackes, die also trotz des Suggestionsversuches der bei Grün sonst eintretenden Sinnessteigerung entsprach. Eine andere Versuchsperson vermochte aus der Art der Einwirkung auf die verschiedenen Sinnesempfindungen und aus der Stärke dieser Einwirkung die Farbe zu erschliessen, mit der die Haut bestrahlt wurde.

Besonders erwähnenswert scheint mir folgender Fall zu sein: Bei einer Versuchsperson trat, während sie durch ein blaues oder rotes Glas durchsah, an der mit Zucker bestrichenen Zungenspitze ein Brennen auf, bei Blau stärker als bei Rot; dabei zeigt sich an der Zungeuspitze ein schwacher bitterer Geschmack. Grün erregte ebenfalls ein Brennen, gleichzeitig aber eine auffällige Änderung der Geschmacksempfindung Süss in Bitter. Ganz dieselbe Erscheinung bot eine Finwirkung des farbigen Lichtes auf die seitlichen Halsund Gesichtspartien dar. Bei sorgfältigst vorgenommenem Augenverschlusse erkannte die Versuchsperson, bei den wiederholt angestellten 
Versuchen und Demonstrationen jedesmal mit Bestimmtheit in den Auftreten eines stark bitteren Geschnackes an der Zungenspitze die Einwirkung des grünen Lichtes auf die Haut; während sie in dem Erscheinen eines einfachen Brennens auf der Zungenspitze, ohne oder mit nur sehr schwachem bitteren Geschmack, stets richtig auf Blau oder Rot schloss.

Wenngleich ich nicht glaube, dass mir bei diesen zuletzt besprochenen Versuchen eine Täuschung unterlief, so ist doch diesen Beobachtungen: gegenüber die grösste Vorsicht geboten, und eine Klarstellung dieses Gegenstandes bleibt erst weiteren, eingehenden Untersuchungen vorbehalten.

Bei einem Versuche über den Einfluss der Farbenempfindungen auf die Sinnesfunctionen beobachtete ich, dass auch gewissë Körperbewegungen und -stellungen einen Einfluss auf die Stärke der Sinnesempfindungen zu nehmen vermögen. Ich wurde darauf durch einen Fall aufmerksam gemacht; wo bei Prüfung mit einer vor die Augen gehaltenen farbigen Glastafel regelmässig eine auffällig stärkere Reizung der verschiedenen Sinnesempfindungen entstand, wenn die betreffende Versuchsperson die: Glastafel mit beiden Händen oder mit einer Hand zur Augenhöhe emporhob, als wenn dies durch eine andere Person gesehah, während die Versuchsperson die Hände in gesenkter Stellung hatte. Als jeh ohne Farbeneinwirkung die Arme heben liess, zeigte sich, dass durch das Erheben der Arme allein eine Steigerung der verschiedenen Sinnesempfindungen erfolgte, die so lange anhielt, als die Armerhebung währte, auch wenn diese passiv stattfand. Mit dem Senken der Arme trat wieder eine Schwächung der Sinnesempfindung auf die frühere Stufe ein. Hebungen der unteren Extremitäten, stärkere Neigung des Kopfes, Compression der Carotis am Halse riefen ähnliche Steigerungen der Sinnesempfindungen hervor wie das Erheben der Arme. Untersuchungen, die ich darüher mit anderen Personen anstellte, zeigten, dass diese Erscheinung häufig besteht; aber in einer individuell sehr verschiedenen Weise ausgeprägt ist. Auch die Localisation des subjectiven Hörfeldes sowie die von subjectiven Hörempfindungen kann durch die angeführten Körperbewegungen beeinflusst werden. So ergab in einem Falle ein beiden Ohren durch Hörschläuche zugeleiteter Ton ein in der rechten Hinterhauptshälfte gelegenes subjectives Hörfeld; beim Heben der linken Hand zeigte sich ein solches auch in der linken Hinter- 
Über den Einfluss der Farbenempfindungen auf die Sinnesfunctionen. 119

hauptshälfte, das beim Senken des linken Armes wieder schwand. Beim Heben des rechten Arms rückte das subjective Hörfeld von der rechten Hinterhauptshälfte ins rechte Ohr und beim Senken des rechten Arms wieder gegen das Hinterhaupt. Da meinen früher mitgeteilten Beobachtungen zufolge die Wanderung des subjectiven Hörfeldes aus "der Kopfmitte in das Ohr auf einer ansteigenden Hörempfindung dieses Ohres berubt, so deutet demzufolge das Wardern des subjectiven Hörfeldes aus der Hinterbauptgegend in das rechte Obr beim Erheben der. rechten Hand auf eine dabei erfolgende Steigerung der Hörempfindung: im rechten Ohre hin. Ein Erheben beider Arme ergab in diesem Falle eine verstärkte Wahrnehmung des subjectiven Hörfeldes in der rechten Hinterhauptshälfte.

Die durch Erheben der Arme eintretende Sinnessteigerung kann auf einer Seite stärker ausgeprägt sein als auf der anderen. In einem Falle, wo in beiden Ohren die subjectiven Gehörsempfindungen von pulsierendem Wassergeräusche und von Seidenrauschen bestanden, wurde beim Erheben beider Arme das Wassergeräusch nur im rechten Ohre verstärkt.

Die geschilderten Körperstellungen können auch eine qualitative Änderung der Sinnesempfindungen veranlassen; so erfolgte bei dem S. 106 erwähnten Collegen, bei dem durch verschiedene Farben eine süsse Geschmacksempfindung in eine bittere umgewandelt wurde, auch beim Erheben der Arme ein bitterer Geschmack an den mit Zucker bestrichenen Zungenrändern; beim Senken der Arme trat wieder der süsse Geschmack hervor. 